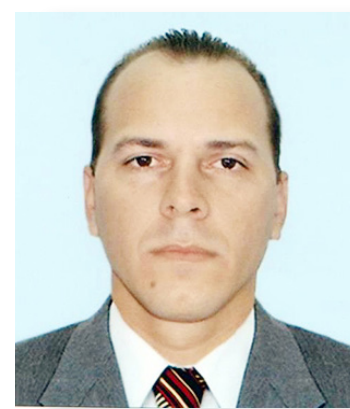

Dr. Rafael Hernández

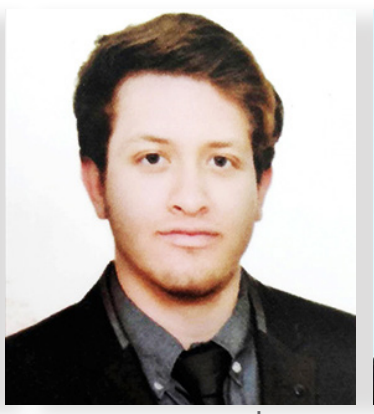

Est. Francisco Rivadeneira²

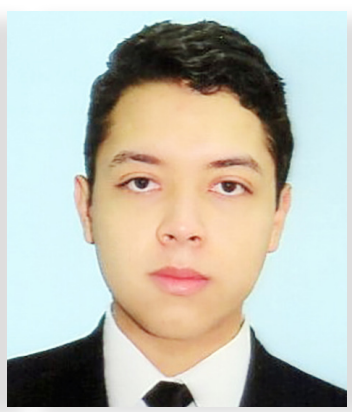

Est. César Galeano ${ }^{3}$

\title{
Herramientas informáticas de apoyo a la redacción del texto científico
}

\section{Computer tools to support the writing of the scientific text}

Recibido: 18-09-18

Aprobado: 02-01-19

\section{Resumen}

El investigador escribe en un informe la ciencia que produce; sin embargo, esta práctica no es suficiente para dominar la redacción del texto científico; atendiendo a ello, este estudio se propone como objetivo mostrar recursos informáticos que pudieran favorecer la labor de estudiantes durante la redacción de sus textos científicos. Se ha desarrollado siguiendo la perspectiva cualitativa, centrado en la descripción, la interpretación y la comprensión de la situación que se investiga, a partir de entrevistas, grupos focales y evaluación con rúbrica a los textos producidos por los estudiantes involucrados en la misma. El estudio se llevó a cabo con los estudiantes, seleccionados intencionalmente, de primer semestre de las carreras de Ingeniería en Software, Licenciatura en Derecho y Licenciatura Administración de Empresas y Negocios, que se imparten en la Universidad Regional Autónoma de los Andes (UNIANDES), extensión Ibarra, Ecuador, durante el período octubre de 2017 a febrero de 2018. Como resultado, se exponen recursos que contribuyen a la redacción científica; se concluye que su utilización adecuada mejora el producto textual de los estudiantes cuando afrontan la elaboración del proyecto integrador.

Dr. Rafael Carlos Hernández Infante, PhD: Doctor en Ciencias Pedagógicas. Profesor - investigador de la Universidad Regional Autónoma de los Andes (UNIANDES), extensión Ibarra, Ecuador. Autor y coautor de libros y artículos en revistas indexadas. Para contactar al autor: rafaelcarlos_docente@outlook.com

${ }^{2}$ Est. Francisco Rivadeneira Enríquez: Estudiante de la carrera de Ingeniería en Software de la UNIANDES, extensión lbarra, Ecuador. Alumno ayudante de la materia Metodología de la investigación. Para contactar al autor: rivadeneirafrancisco21@gmail.com

${ }^{3}$ Est. César Josué Galeano Páez: Estudiante de la carrera de Ingeniería en Software de la UNIANDES, extensión lbarra, Ecuador. Alumno ayudante de la materia Metodología de la investigación. Para contactar al autor: cjosuegp@hotmail.com

${ }^{4}$ Est. Marcos Andrés Rosero Bolaños: Estudiante de la carrera de Ingeniería en Sistemas de la UNIANDES, extensión lbarra, Ecuador. Para contactar al autor: markorosero.nayan@gmail.com

${ }^{5}$ Dra. María Elena Infante Miranda, PhD: Doctora en Ciencias Pedagógicas. Profesora - investigadora de la UNIANDES, extensión Ibarra, Ecuador. Autora y coautora de libros y artículos en revistas indexadas. Para contactar a la autora: m_infante_docente@hotmail.com 


\section{2 • ARTí́CULOS CIENTÍFICOS}

\section{Abstract}

The researcher writes in a report the science he/she produces; however, this practice is not sufficient to know the writing of a scientific text; based on this, this research aims to expose computer resources that favor the work of the researcher during the writing of the scientific text. It has been developed following the qualitative perspective; it has focused on the description, interpretation and understanding of the situation being investigated, based on the subjects involved in it. The study was achieved with the students, intentionally selected, in the first semester of the Software Engineering, Bachelor of Law and Bachelor of Business Administration and Business, which are taught at the Autonomous Regional University of the Andes (UNIANDES), extension Ibarra, Ecuador, during the period October 2017 to February 2018. As a result, resources that contribute to scientific writing are exposed; it is concluded that its proper use favors the preparation of students when they face the elaboration of the integrating project.

\section{Palabras clave}

recursos; redacción; texto científico; investigador; ordenador

\section{Keywords}

resources; writing; scientific text; researcher; computer

\section{Introducción}

Dentro de las etapas que conforman el método científico, se señala la correspondiente a la publicación de sus resultados (Estévez, 2006; Córdova, 2014; Hernández e Infante, 2018; Salazar, 2018); para lograr este propósito, resulta necesaria la difusión del conocimiento a través de libros, ponencias, revistas de impacto, entre otras formas; por ello, ante el investigador se presenta la necesidad de redactar el texto científico haciendo un uso adecuado de estrategias discursivas y utilizando, a la vez, herramientas que puedan mejorar y facilitar esta labor.

Este tipo de texto pertenece a la formación funcional estilística de trabajo profesional (Dubsky, 1983; Armas, Pérez y Rivero, 2017), de ahí sus particularidades. Su propósito esencial es la comunicación de un resultado científico; requiere un plano semántico uniforme, el empleo de términos exactos y automatismos definidos o codificados, propios de un sector del saber; es decir, exige la utilización de palabras de significación exacta y unívoca, el predominio de lo denotativo; el uso de tecnicismos, cientifismos y extranjerismos (Alpízar, 1990; Grass, 2002; Cassany, 2007; Rodríguez, 2009; Roméu, 2012; Ávila, 2017 y Gené, Olmedo, Pascual, Azagra, Elorduy y Virumbrales, 2018); las particularidades apuntadas indican que su redacción es compleja.

Es una realidad que el investigador tiende, por oficio, a escribir la ciencia que produce; sin embargo, muchas veces esta práctica no le resulta suficiente o fácil, por ejemplo, cuando debe mostrar un poder de síntesis elevado durante la redacción del texto científico. Cabe resaltar que cuando se habla de investigadores "en ciernes", como es el caso de estudiantes que asumen por primera vez la actividad de indagar desde la ciencia, la referida dificultad se acrecienta.
La experiencia de los autores de este texto en la asesoría de trabajos investigativos estudiantiles, en las carreras que se imparten en la Universidad Regional Autónoma de los Andes (UNIANDES), extensión Ibarra, Ecuador, que constituyen la población de la presente investigación, les permite detectar deficiencias en la elaboración del texto científico en el cual plasman los resultados obtenidos en sus proyectos. Las insuficiencias que se evidencian están dadas, en lo fundamental, en el uso de estructuras gramaticales mal construidas, la repetición de palabras y en la no aplicación de algunas reglas ortográficas, lo que afecta la comunicación de las ideas; esta situación incide en que para los alumnos sea una tarea engorrosa la redacción de su proyecto integrador.

El proyecto integrador es un trabajo de investigación, estipulado en la malla curricular, que integra el contenido de las diferentes materias que reciben los estudiantes en cada nivel de su carrera. Al finalizar cada período académico, los educandos presentan el resultado de la investigación desarrollada en un texto que sintetiza el proceso de indagación científica y que debe cumplir con normas de redacción establecidas para este tipo de texto, lo que complejiza el trabajo a ejecutar por los estudiantes.

En la actualidad existen medios, materiales, recursos, herramientas que pudieran contribuir de manera eficiente a resolver en parte la dificultad expresada. Cabe destacar, que más allá de esta polisemia mostrada, se desea hacer referencia a un elemento de carácter didáctico que "(...) intencionalmente utilizamos para conseguir el objetivo que se pretende; en este caso, desarrollar eficaz y eficientemente los procesos didácticos de enseñanza y de aprendizaje (..)" (Castillo y Cabrerizo, 2006, p. 260). Atendiendo a lo declarado, 
este artículo se propone mostrar recursos informáticos que pudieran favorecer la labor de todos los que tienen que llevar a cabo la redacción de un texto científico. En aras de su cumplimiento se desarrollan todas las acciones posteriores.

Es evidente que la computadora juega un papel fundamental en el proceso de escritura de la ciencia. En la actualidad es difícil imaginar al investigador, en plena labor, alejado de este dispositivo electrónico. En sentido general, aunque se reconoce que existen otras clasificaciones sobre el uso del ordenador, se asumen, por su alcance, las siguientes direcciones (Hernández, 2012):

- Como objeto de estudio: su empleo va encaminado al uso de las diferentes aplicaciones y programas, así como el propio sistema operativo. Es decir, este enfoque apunta a la operación del ordenador por parte de un usuario específico.

- Como medio de enseñanza-aprendizaje: este uso revela las potencialidades del dispositivo electrónico para "aprender de él y con él"; mediante su empleo se hace más vívida la adquisición de los conocimientos por parte de los estudiantes.
- Como herramienta de trabajo: se enfoca esta perspectiva en el empleo del ordenador haciendo uso de las aplicaciones y programas, de acuerdo a la selección que haga el usuario.

Este último uso específico engloba de manera más directa el trabajo del investigador durante el proceso de escribir la ciencia que produce. Por este motivo se privilegia en el presente artículo el uso de la computadora como herramienta de trabajo del investigador.

Cabe destacar que hoy día no resulta una novedad afirmar que durante el proceso de redacción con fines investigativos se hace uso de un procesador de textos, el cual no es más que una aplicación para crear y manipular documentos. Existe una gama de sistemas operativos con procesadores de texto diseñados para los ordenadores personales. Los mismos se dividen en gratuitos y de pago.

Entre los procesadores gratuitos de texto de escritorio, se hace referencia a la suite ofimática OpenOffice. Esta ofrece variadas opciones; en contraposición a ella, como parte de los programas de pago se encuentra, como uno de los más difundidos, el Microsoft Office (Hernández, 2011; Hernández, Infante y Córdova, 2016).

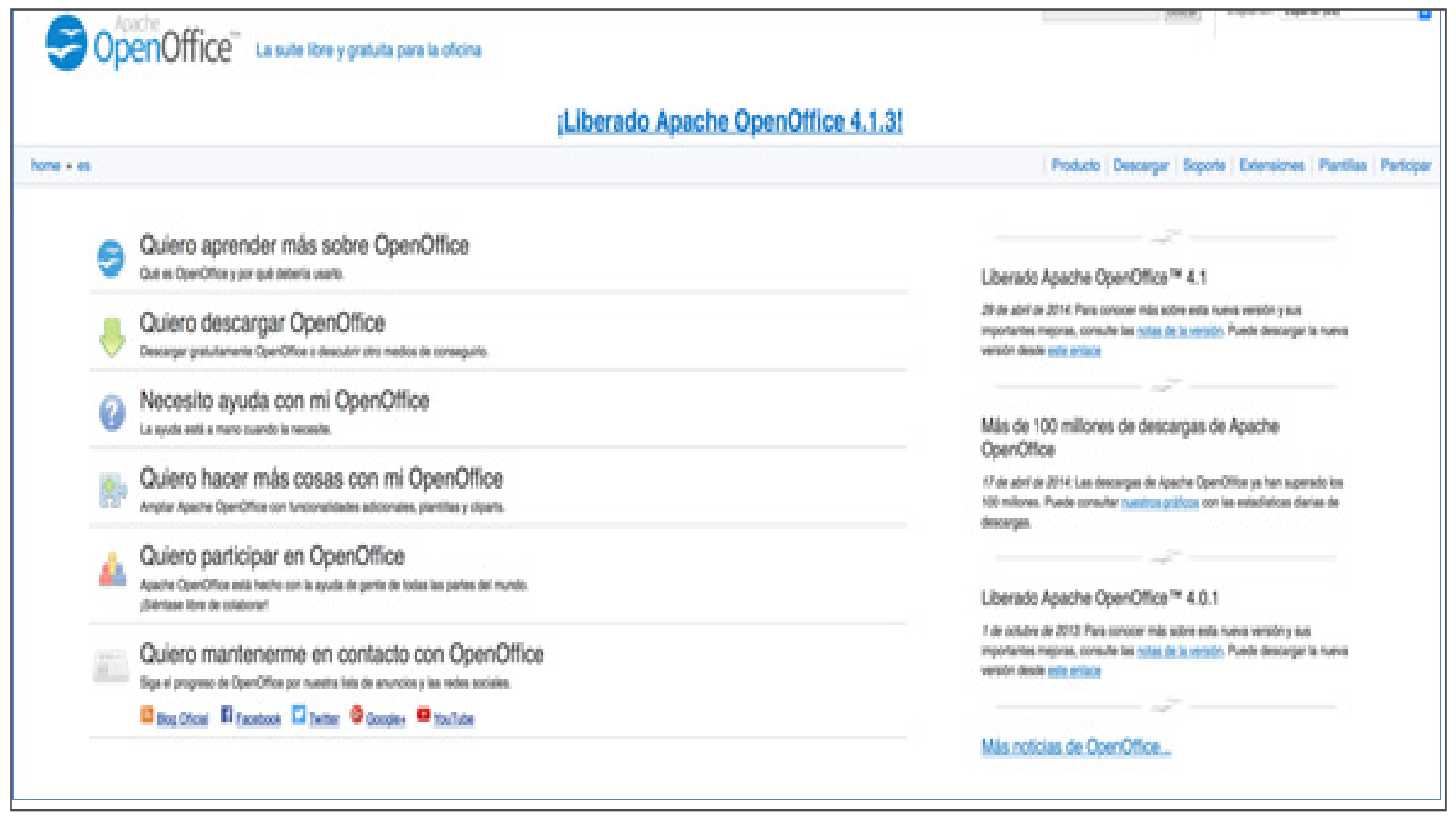

Figura 1: Suite ofimática OpenOffice. Fuente: https://www.openoffice.org/es/

En relación con lo expresado, debe apuntarse que en la actualidad la suite de oficina antes mencionada posee un atributo particular. Este es aportado por el Cloud computing: "(...) aplicaciones que actúan como servicios basados y utilizados exclusivamente en internet. (...) no es necesario -para su uso- la instalación de ninguna aplicación o software de manera local en nuestro ordenador" (Peña, 2013, p. 184). De esta manera, pueden encontrarse las versiones: "Office 365 Hogar Premium" (de pago) y como alternativa: "Open365" (gratuita). 


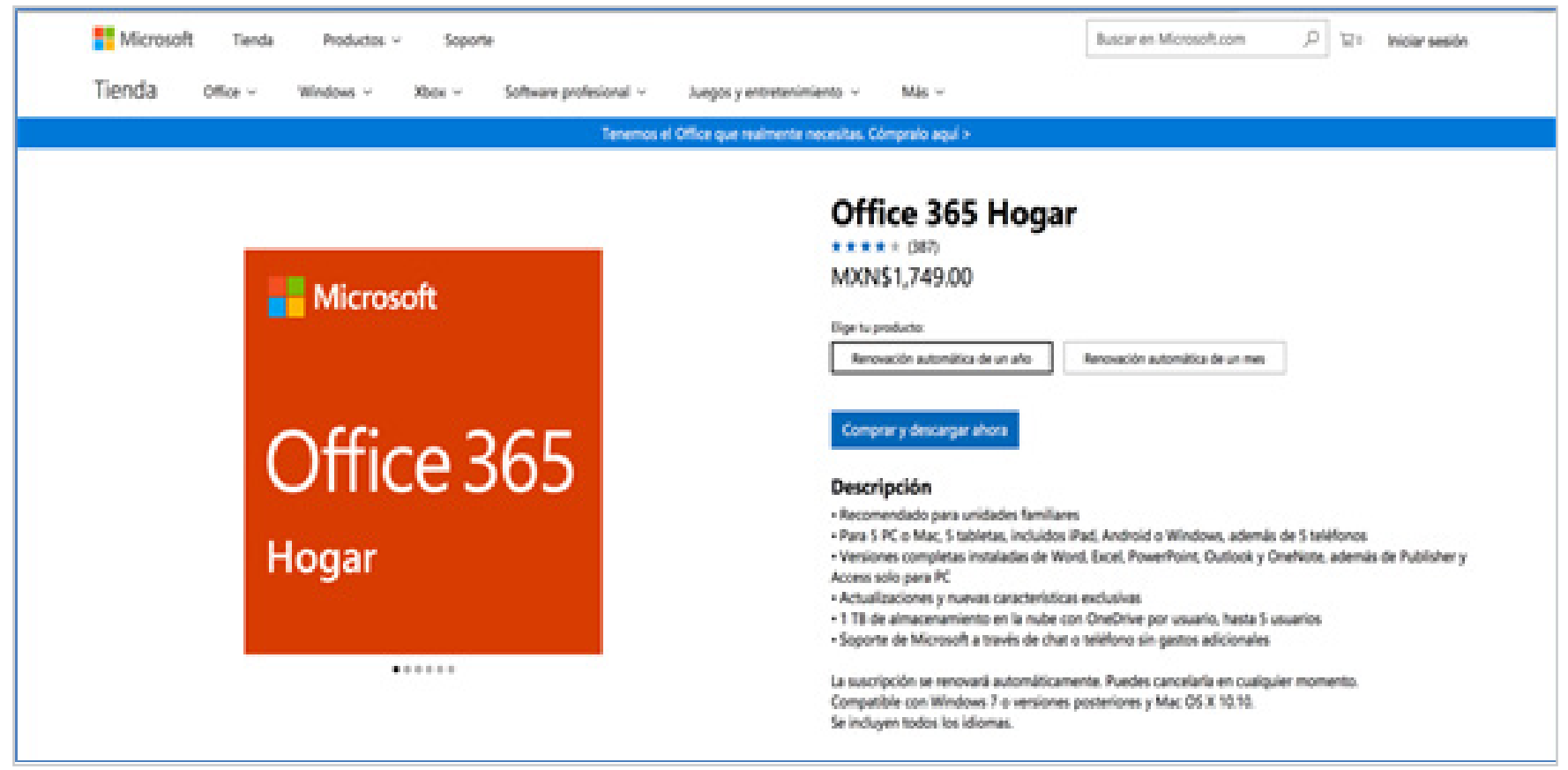

Figura 2: El Office 365 Hogar Premium que ofrece Microsoft. Fuente: https://products.office.com

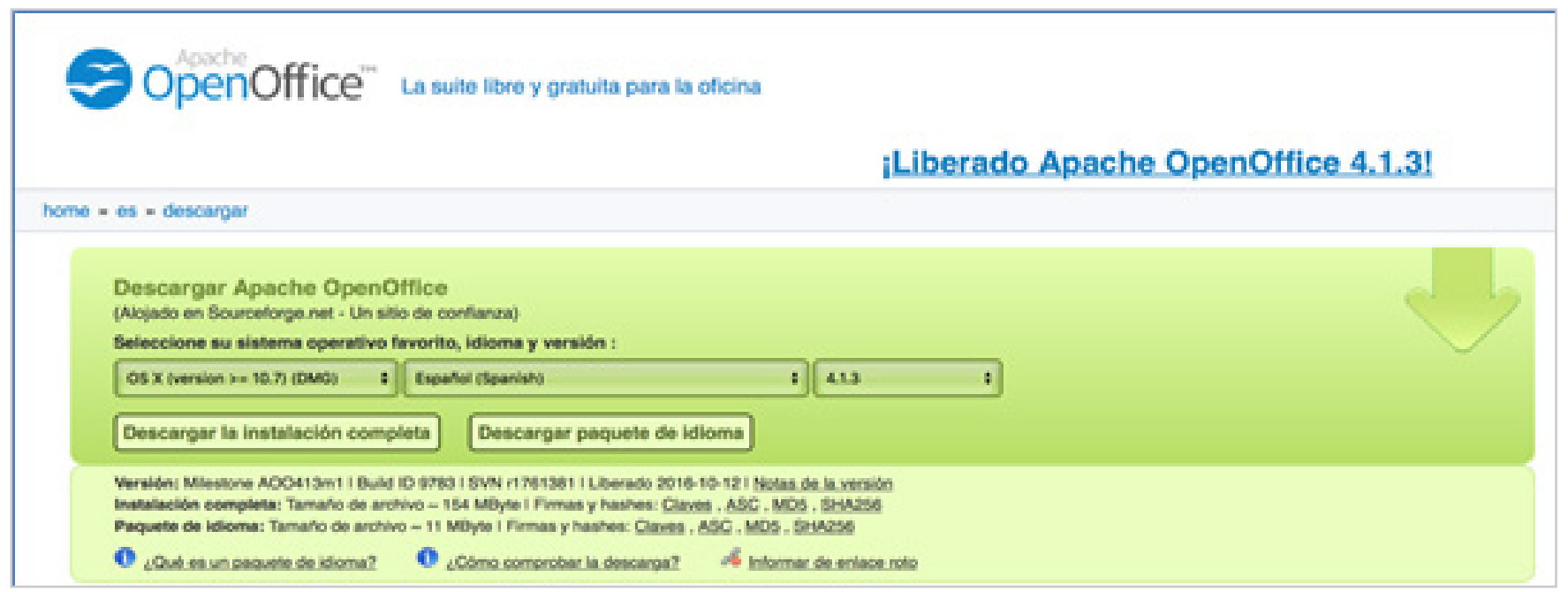

Figura 3: El Open365 basado en Open Source. Fuente: https://www.openoffice.org/es/descargar/

Por otro lado, se debe tomar en cuenta que en el proceso de escritura se incurre muchas veces en fallos en la normativa de la gramática, por lo tanto, se necesita de una revisión y ayuda permanente para la corrección. Si bien es cierto que los procesadores de textos ofrecen esta opción, existen herramientas que se ofrecen en línea, cuyo empleo resulta orientador en la etapa de redacción y edición del texto.

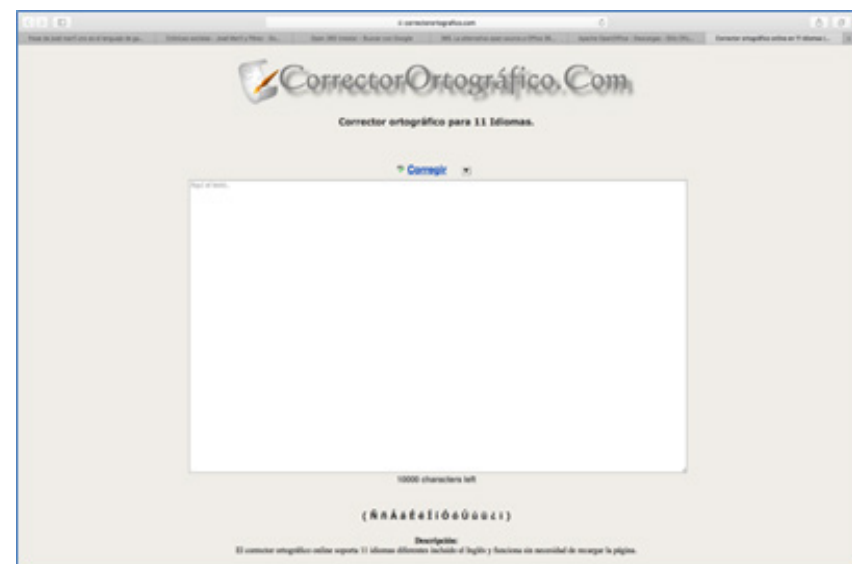

Figura 4: Ejemplo de corrector ortográfico en línea. Fuente: https://www.correctorortografico.com/ 
A través de un corrector ortográfico, como el que se muestra en la imagen anterior, se accede a recursos de corrección, con una aceptación de textos escritos hasta en 11 idiomas. A propósito del carácter automatizado del proceso que se genera, no debe hacerse a un lado el hecho de que es el autor o los autores los que deben tomar partido y asumir de manera protagónica la toma de decisión en relación a las sugerencias que reciben del software. De igual forma, es importante el dominio del código escrito para alcanzar una mayor eficiencia y claridad (Hernández, 2011; Hernández, Hernández y Terrazas, T. 2015; López y Eliza, 2018).

Los diccionarios constituyen otras herramientas de utilidad para una redacción adecuada; los que escriben en idioma español tienen a su alcance el Diccionario de la Lengua Española (Real Academia Española, 2014). Aparejado a esto, se hace referencia a uno con un nivel más alto de especialización: el Diccionario Panhispánico de Dudas (Real Academia Española, 2017). Su finalidad está relacionada con aclarar dudas léxicas sobre el uso de determinados vocablos, así como también sobre el empleo de neologismos.

Cabe destacar que, para una rama específica de la ciencia, como lo es el Derecho, por ejemplo, puede encontrarse el Diccionario del español jurídico (Real Academia Española, 2016). Por otro lado, aparece en línea una variada gama de diccionarios que abordan más de siete categorías diferentes del saber.

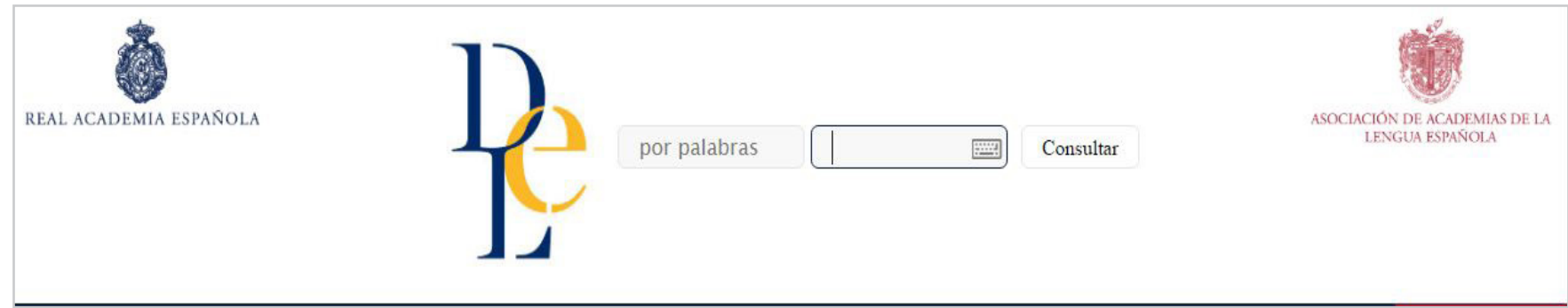

Figura 5: Ejemplo de diccionario en línea: Real Academia Española. Fuente: http://dle.rae.es/?w=diccionario

Escribir la ciencia demanda, entre otros aspectos, el no incurrir en faltas que apuntan directamente a las propiedades del texto como la coherencia y la cohesión, que afectan a la construcción del texto. El investigador debe ejercitar la etapa de revisión de su texto y descubrir ideas cuya estructura pudiera resultar confusa. También, puede utilizar diccionarios que le permitan buscar información para sustituir determinados vocablos de manera que se eviten repeticiones innecesarias en el texto. Hacer uso de un diccionario de sinónimos y antónimos contribuye a ese fin, aunque no se hace a un lado el hecho de que los procesadores de textos también ofrecen ese recurso (Hernández, 2011); sin embargo, en línea se encuentran recursos alternativos, como es el caso del Buscador de ideas afines y relacionadas.

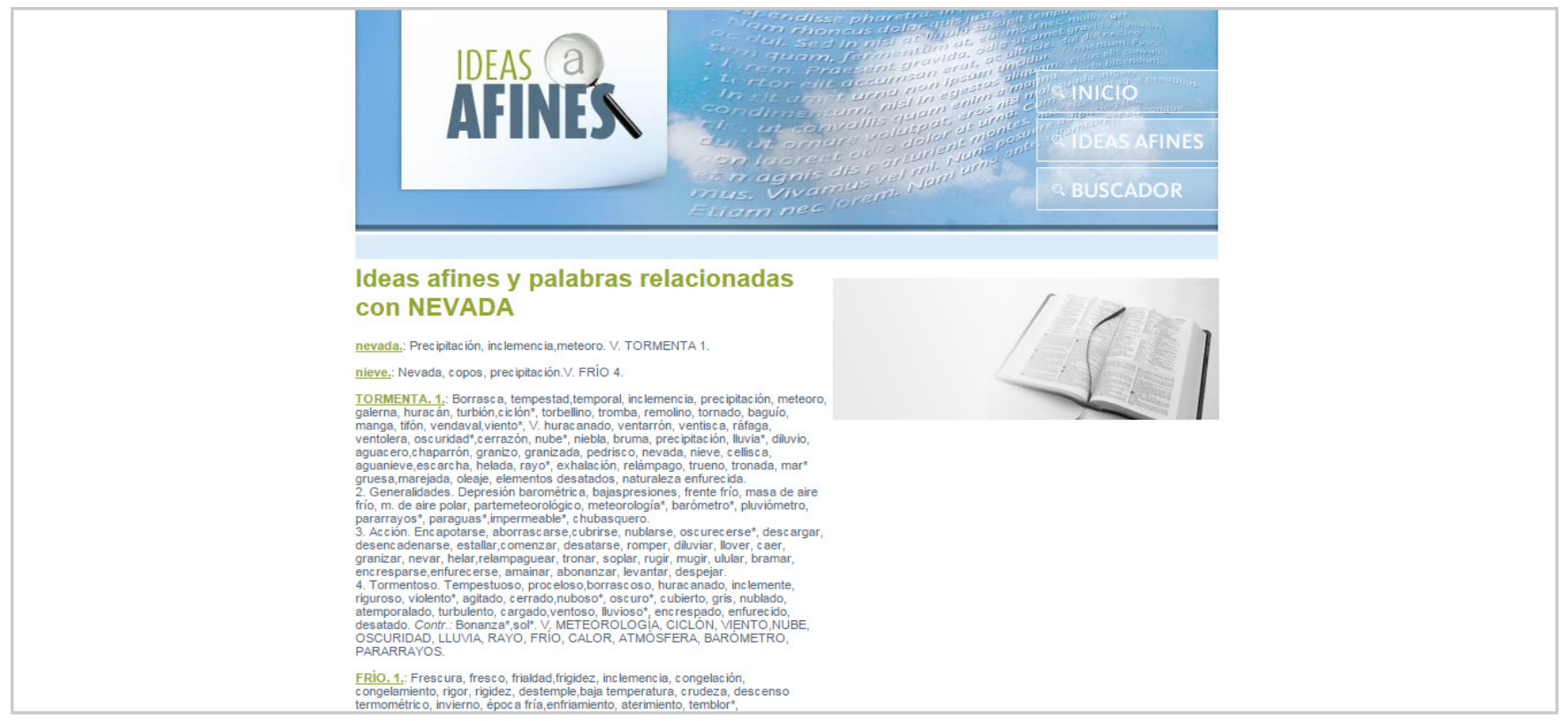

Figura 6: Buscador de ideas afines y relacionadas, en línea. Fuente: http://www.ideasafines.com. ar/buscador-ideas-relacionadas.php 
No debe perderse de vista, por su utilidad, el empleo de los glosarios (catálogos de palabras pertenecientes a una disciplina o a un campo de estudio). Los vocablos que aparecen en ellos requieren ese apoyo para su entendimiento (Bustos, 2009), pues en este tipo de documento se definen, se explican o se comentan, siempre ordenados de forma alfabética, para que se comprenda su significado en un contexto específico (López, 2004; Delis, Campaña-Jiménez y GallegoArrufat, 2018).

Dentro de los glosarios que pueden ser utilizados por los investigadores, se cita como ejemplo el "Catálogo de palabras de una misma disciplina (...) definidas o comentadas" (RAE Gestión, 2016). Su uso contribuye, en particular, a enriquecer el léxico del investigador cuando escribe sobre algún tema especializado. De la variedad de estos recursos que aparecen en línea se sugiere, por su nivel de generalidad, consultar el de expresiones coloquiales, lo que puede ayudar al investigador a tomar conciencia sobre aquellos términos que deben evitarse en un trabajo de carácter científico.

\section{Coloquialmente}

Clossio espaisal de andar por cass

nicio Acerade Calegorias Contacur

\section{AIBICIDIEIFIGIHIIIJKILIMINIOIPIOIRISITIUIVIIIZ}

Figura 7: Buscador de expresiones coloquiales, en línea. Fuente: http://www.coloquial.es

Como se ha dejado entrever con antelación, el investigador está comprometido con la publicación de los resultados de su estudio. Sin esta acción no estaría completo su trabajo ni cerraría el ciclo que demanda la aplicación del método científico; por ello, redactar el informe de la investigación es fundamental. Sin embargo, no debe perderse de vista que esta labor requiere seguir pautas específicas, atendiendo a las particularidades del texto científico. En este sentido, juegan un importante papel los manuales relacionados con el estilo científico o profesional; se sugieren, por su amplitud, fundamentos y carácter didáctico, los siguientes: el "Diccionario urgente de estilo científico del español" (Pérez, 1999) y el "Libro de estilo de ABC" (Vigara, 2001). Los recursos referidos son esenciales para el investigador que se decida a publicar los resultados de su trabajo en la lengua castellana.

Eah

Ninghin oblook disponible

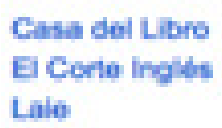

Lilp

Duscar an uni biblokst

Todos kos wondudaris :
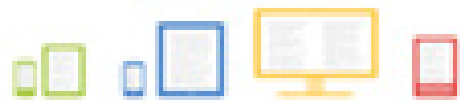

\section{Libro de estilo de $\mathrm{ABC}$}

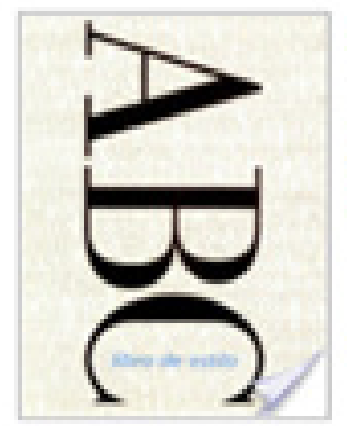

Ma Marla Ygara Taushe

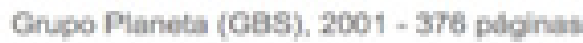

the $\begin{aligned} & \text { in } \\ & \text { int }\end{aligned}$

D Rosolnas.

6.

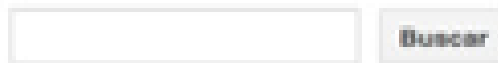

Vata presla dell libro :

Figura 8: El libro de estilo de ABC, en línea. Fuente: https://www.abc.es 


\section{DICCIONARIO URGENTE DE ESTILO CIENTÍFICO DEL ESPAÑOL}

\author{
Juan Antonio Pérez Ortiz \\ japerez [en]dlsi.ua.es \\ Marzo 1999
}

Figura 9: Diccionario urgente de estilo científico del español, en línea. Fuente: http://www.dlsi.ua.es/ japerez/pub/pdf/ duece.pdf
Por otra parte, Grammarly es una alternativa para la redacción científica. Funciona como extensión en el navegador y también como aplicación de escritorio de acuerdo a los permisos del usuario de la aplicación. Grammarly corrige errores ortográficos y de redacción mientras hace cualquier tipo de tarea. Se encuentra esta aplicación gratis o de pago.

Otra de las herramientas de alcance y utilidad es el Google Docs. Esta consiste en un editor de texto integrado dentro de Google Drive. Permite compartir el mismo documento con varios usuarios a la vez. Soporta una variedad de idiomas y ofrece la posibilidad de añadir comentarios o modificaciones al texto.

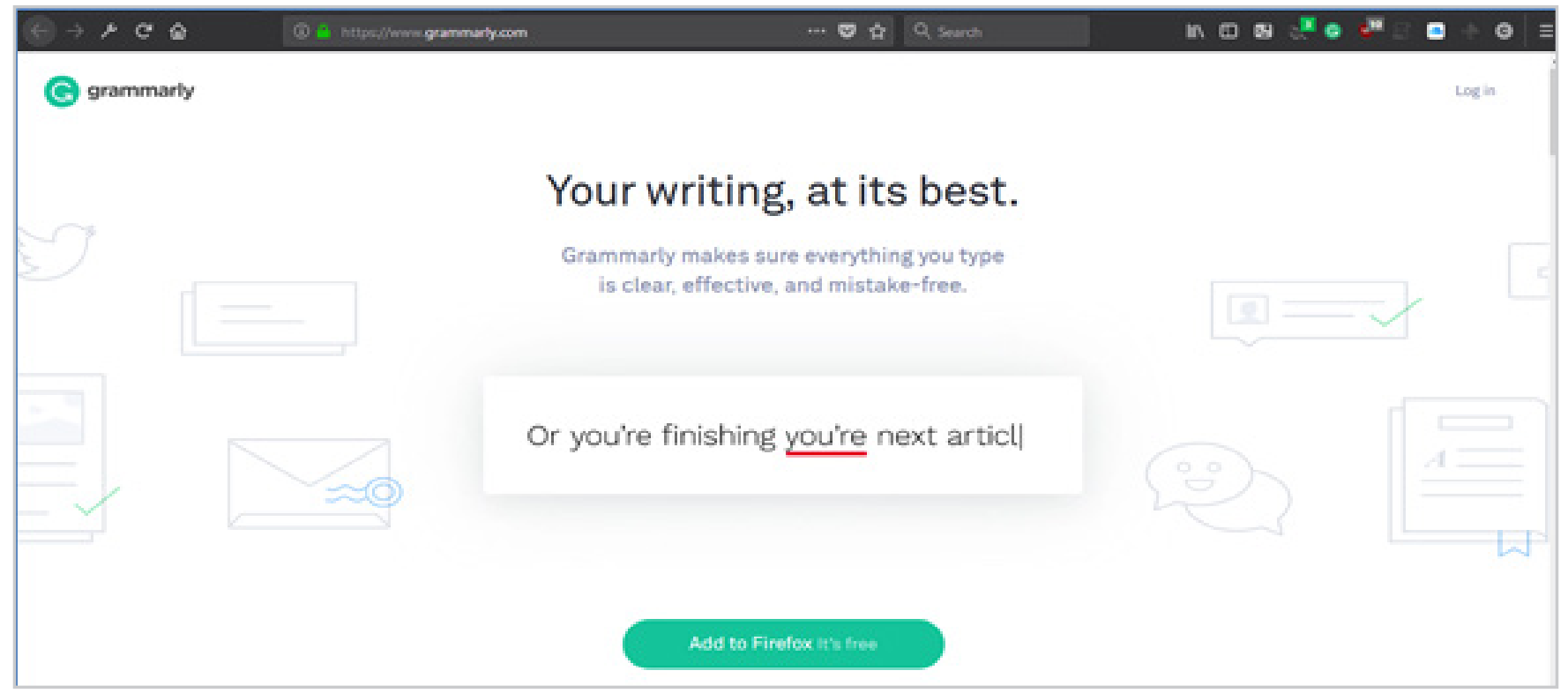

Figura 10: Entorno de la aplicación Grammarly. Fuente: https://www.grammarly.com

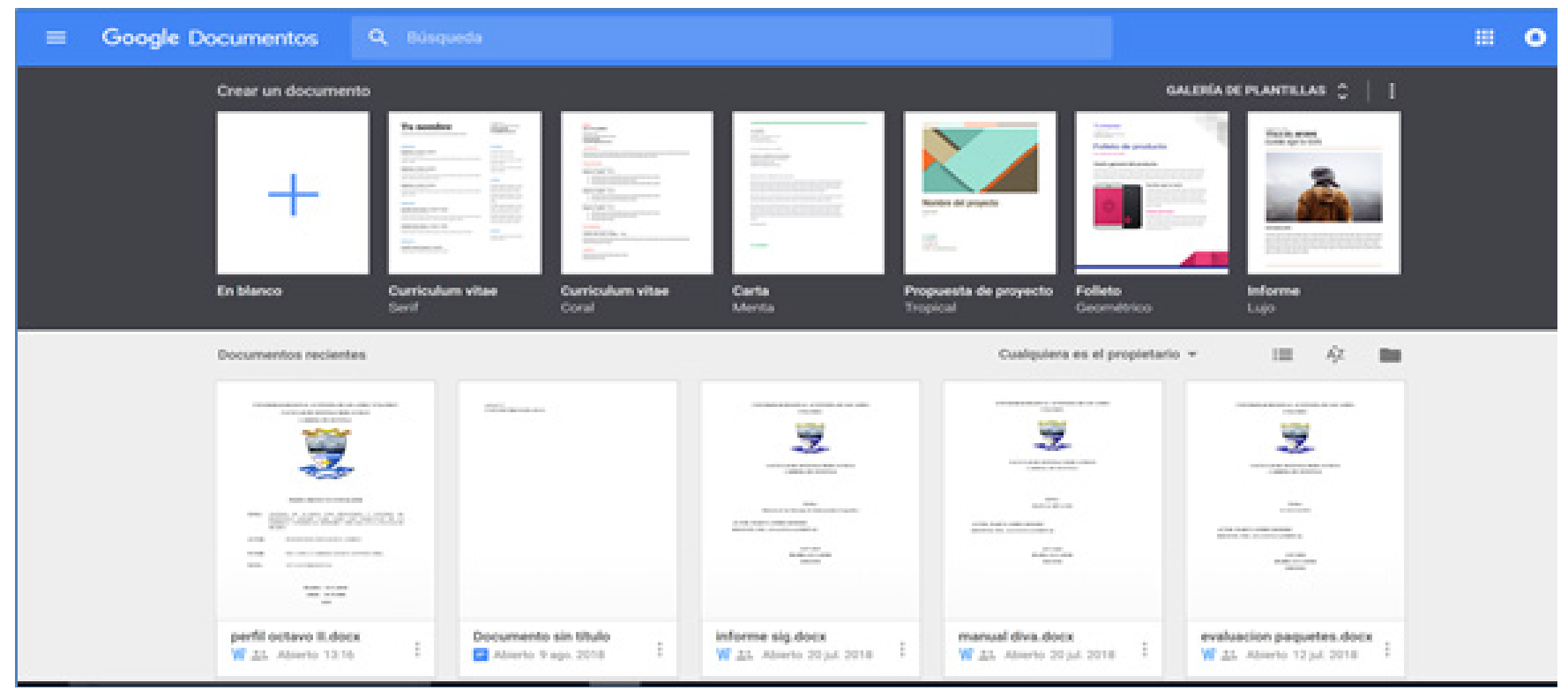

Figura 11: Entorno del Google Docs. Fuente: https://www.google.com 


\section{Metodología}

La investigación se ha desarrollado siguiendo las pautas de la perspectiva o modalidad cualitativa; se ha centrado en la descripción, la interpretación y la comprensión de la situación que se investiga, a partir de los sujetos involucrados en el mismo. Se escogió la muestra de manera intencional (no probabilística), tomando en consideración la relación estrecha de los autores con los alumnos seleccionados y el hecho de que estos últimos estaban desarrollando su proyecto integrador durante el período que se investiga: octubre 2017-febrero 2018.

Desde el nivel empírico se emplea la entrevista. La misma se clasifica como cualitativa y no estandarizada; se parte de una guía como instrumento flexible, abierto, útil para estudiar el objeto, permitiendo a los sujetos manifestar sus criterios libremente. Desde su aplicación se obtienen las evidencias que sustentan las dificultades existentes en el objeto de investigación. En línea con el objetivo declarado, se procedió a entrevistar a 37 estudiantes de primer semestre de las carreras de: Ingeniería en Software, Licenciatura en Derecho y Licenciatura Administración de Empresas y Negocios.

A la muestra seleccionada se le aplicó una entrevista durante tres momentos en el desarrollo del semestre (Ver Anexo 1). Debe expresarse que fue necesario introducir nuevos aspectos a cada entrevista, pues las respuestas obtenidas en una ronda indicaban la necesidad de ahondar en otros elementos que evidenciaban las necesidades y debilidades que caracterizaban los textos que estaban produciendo los estudiantes. Dichas entrevistas arrojaron la valoración de los estudiantes sobre las competencias escriturales que consideraban necesarias para la producción del proyecto integrador.

El análisis del contenido de las respuestas de los entrevistados permitió identificar las deficiencias. Estas se muestran a continuación, atendiendo a las diferentes preguntas en cada entrevista.

\section{Primera ronda:}

- Se evidencia desconocimiento sobre el objetivo que se persigue con la realización del proyecto integrador del nivel.

- Existen dudas sobre las temáticas a abordar y su relevancia.

- Tienen escasa visión acerca del alcance de la investigación y el contexto de desarrollo.

\section{Segunda ronda:}

- Afloran dudas en relación con la estructura que debe seguir el proyecto integrador como documento en el que plasmarán los resultados investigativos que alcancen.

- Hay dudas en relación con la integración de los aspectos de la investigación en desarrollo y el contenido de las materias que reciben.

\section{Tercera ronda:}

Cabe destacar que como paso previo a esta ronda se procedió a realizar una revisión del trabajo en elaboración. Ello, juntamente con la entrevista, permitió obtener la siguiente información:

- Se nota dispersión en relación con el tratamiento de la información recopilada, tanto teórica como práctica.

- Existe dificultad en relación al poder de síntesis a mostrar durante el desarrollo del informe.

- Se valoran como "difíciles y de bajo dominio" las normas de la redacción científica que deben aplicar en la presentación del proyecto. En particular, los textos carecen de: objetividad, impersonalidad, precisión en el empleo de términos, predominio de lo denotativo; precisión y gramaticalidad en el dominio de la normativa de la lengua escrita; coherencia y cohesión en las ideas expresadas.

La información que han ofrecido estas entrevistas, así como la valoración del trabajo escrito, indica la necesidad de utilizar la técnica de tormenta de ideas para conocer las posibles soluciones a las dificultades detectadas; del intercambio surgieron dos directrices fundamentales:

- Realizar un taller sobre las normas de la redacción científica que deben aplicar en la presentación del proyecto, de modo que pudiera profundizarse en las mismas desde la teoría y desde la práctica, a través de ejemplos concretos.

- Identificar herramientas informáticas que ayuden a los estudiantes en el proceso de redacción y edición del texto que presentarán como proyecto integrador, por ejemplo, aquellas que les permitiesen corregir errores ortográficos y gramaticales; buscar sinónimos, entre otras.

Seguidamente se procedió a planificar el taller. Para el desarrollo de este se realizaron encuentros teórico prácticos en los cuales participaron los estudiantes. Se trabajó dos semanas en el mes de diciembre 2017 y dos en el mes de enero 2018, tiempo que tenían planificado 
los alumnos para redactar el marco teórico de su proyecto. Se laboró: lunes, miércoles y viernes durante dos horas. Se emplearon las computadoras personales de los propios estudiantes. El sistema de trabajo estuvo guiado por la colaboración en equipo. Durante los encuentros se reforzó el proceso de redacción, sugiriendo emplear recursos informáticos como los que se presentan en este estudio. En la etapa final, se les animó a identificar herramientas específicas de acuerdo a las debilidades que se les señalaba en las revisiones del proceso de escritura del proyecto integrador. Se aclararon las dudas, percibiendo la evolución paulatina de los textos. Jugó en esto un papel esencial emplear una rúbrica de evaluación facilitada por una colega (Anexo 2) para medir el nivel de las propiedades textuales.

\section{Resultados}

Los proyectos integradores elaborados por los estudiantes, en sentido general, cumplieron su objetivo en tanto las investigaciones desarrolladas establecieron relaciones entre los contenidos que recibieron en las diferentes materias del semestre y en la búsqueda de una solución al problema revelado. Debe señalarse que el uso de las herramientas informáticas mencionadas fue de utilidad en el proceso de redacción del texto, aspecto en el cual se presentaban dificultades.
Los alumnos, haciendo uso de las aplicaciones Grammarly y del Corrector ortográfico detectaron y corrigieron errores de la normativa y de redacción del texto, lo que favoreció la comunicación de las ideas con la precisión y la claridad que exige un texto de carácter científico. Además, el empleo de diccionarios como el de Sinónimos y antónimos, así como El buscador de ideas afines y relacionadas, resultó un medio adecuado para evitar la repetición innecesaria de palabras; los alumnos se percataron del beneficio de estas herramientas en el proceso de edición del texto que producían.

Es preciso destacar que los estudiantes se auxiliaron del Buscador de expresiones coloquiales para evitar el uso de estas en el texto que elaboraban, reconociendo de ese modo la particularidad del texto científico de utilizar un lenguaje técnico y con carácter denotativo en la comunicación de las ideas. Debe acotarse que la elaboración del proyecto integrador implica un aprendizaje colaborativo, pues la investigación se desarrolla por un grupo de alumnos; por ello, durante el proceso de producción y revisión del texto, les fue útil usar la aplicación Google Docs, mediante la cual compartían el documento que elaboraban, incluyendo comentarios y modificaciones que permitían mejorarlo.

Tabla 1: Evolución de la errdicación de las dificultades manifiestas.

\begin{tabular}{|c|c|c|c|}
\hline DIFICULTADES & AL INICIO DEL PROCESO & EN EL PROCESO & $\begin{array}{c}\text { EN LA ENTREGA DEL } \\
\text { PROYECTO }\end{array}$ \\
\hline $\begin{array}{l}\text { Dominio del lenguaje } \\
\text { científico académico } \\
\text { (cohesión lexical) }\end{array}$ & $\begin{array}{l}\text { Se manifiesta gran debilidad con } \\
\text { muchas palabras repetidas y uso } \\
\text { coloquial de la lengua. Se llevan a } \\
\text { cabo entrevistas para descubrir los } \\
\text { errores en el uso variado y adecuado } \\
\text { del vocabulario técnico y cómo pueden } \\
\text { mejorar. }\end{array}$ & $\begin{array}{l}\text { Se llevan a cabo encuentros } \\
\text { paulatinos. Se trabajó dos semanas } \\
\text { en el mes de diciembre } 2017 \text { y } \\
\text { dos en el mes de enero } 2018 \text {. Se } \\
\text { implementó el uso de diccionarios } \\
\text { y otras herramientas como: } \\
\text { Catálogo de palabras de una } \\
\text { misma disciplina, Ideas afines y } \\
\text { Coloquialmente. }\end{array}$ & $\begin{array}{l}\text { Los textos mejoraron } \\
\text { considerablemente en el } \\
\text { uso del vocabulario propio } \\
\text { de sus disciplinas y en la no } \\
\text { repetición, logrando una mejor } \\
\text { cohesión en cuanto al léxico. }\end{array}$ \\
\hline Ortografía & $\begin{array}{l}\text { Se manifiestan grandes debilidades } \\
\text { en el dominio de la normativa de } \\
\text { la acentuación y en la escritura de } \\
\text { palabras. }\end{array}$ & $\begin{array}{l}\text { A partir de la revisión de los textos } \\
\text { y la entrevista de la tercera ronda, } \\
\text { los estudiantes manifiestan que no } \\
\text { dominan las reglas de ortografía } \\
\text { y que las consideran difíciles y } \\
\text { abstractas. }\end{array}$ & $\begin{array}{l}\text { En los encuentros se les } \\
\text { sugiere el uso de Grammarly } \\
\text { y se les anima a utilizar la } \\
\text { herramienta. Se asumen las } \\
\text { reglas de acentuación }\end{array}$ \\
\hline $\begin{array}{l}\text { Coherencia textual y } \\
\text { capacidad de síntesis. }\end{array}$ & $\begin{array}{l}\text { Se manifiestan fuertes debilidades } \\
\text { cuando se llevó a cabo la primera } \\
\text { revisión de los textos. }\end{array}$ & $\begin{array}{l}\text { Se subrayaron las falencias en los } \\
\text { trabajos. Se les motivó a descubrir } \\
\text { sus errores y a través de lluvia } \\
\text { de ideas, se les motivó a que } \\
\text { identificaran las herramientas que } \\
\text { podrían servirles para mejorar las } \\
\text { debilidades en la construcción de } \\
\text { las ideas. }\end{array}$ & $\begin{array}{l}\text { A partir de la tercera ronda, se } \\
\text { trabajó en el estilo de los textos } \\
\text { y en erradicar las falencias. } \\
\text { Se logró que los estudiantes } \\
\text { adquirieran conciencia y } \\
\text { mejoraran su texto académico } \\
\text { científico. }\end{array}$ \\
\hline
\end{tabular}




\section{Conclusiones}

Redactar la ciencia constituye un reto para el investigador, independientemente de la experiencia que posea. A través de esta acción se comunican los resultados de las indagaciones de manera concreta y objetiva. Por ende, expresarse haciendo un uso adecuado de la lengua materna es esencial.

- Se ha comprobado que los estudiantes objeto de investigación manifestaron deficiencias en relación con la elaboración de su proyecto de investigación, particularmente en lo referente a la redacción científica.

- En línea existe una variedad importante de recursos que contribuyen a perfeccionar el proceso de redacción científica. Emplearlos con eficacia es tarea de cada investigador, sin que ello reste su protagonismo en el proceso de elaboración del texto científico.

- La selección de los recursos tratados en este artículo permite conducir de una mejor manera a los estudiantes para afrontar el desarrollo de su proyecto integrador, su elección consecuente incide positivamente en la mejora de las dificultades detectadas en el proceso de redacción de dicho texto.

- En sentido general, es preciso señalar que, aunque se reconoce que el uso de las herramientas indicadas ha ayudado a los estudiantes en la redacción del texto que presentan como proyecto integrador, en un breve lapso de tiempo no se resuelven todas las deficiencias que se han presentado en la elaboración de este tipo de texto. El nivel de dominio de la competencia textual es complejo y depende de diferentes habilidades a desarrollar de manera paulatina; por lo tanto, es menester continuar la labor encaminada a su mejora, tomando en consideración los saberes previos de los alumnos y sus potencialidades para acceder a un estadio superior en una tarea tan importante en sus estudios de pregrado y en su vida profesional.

- No pretendemos ofrecer la última palabra sobre un tema tan complejo y diverso como es la redacción de textos científicos académicos y los recursos en línea que colaboran en su mejoría, sino presentar, fundamentalmente, la facilidad de uso y el beneficio de dichos recursos. Se considera esencial suscitar la curiosidad sobre el tópico tratado, por su importancia para la presentación de los resultados investigativos.

- No debe soslayarse el papel protagónico del investigador en este proceso, la necesaria cultura científica que debe poseer y el adecuado conocimiento de la lengua en la cual se expresa, todo lo cual influye en la calidad del texto que elabora.

\section{Referencias}

Alpízar, R. (1990). Traducción y terminología científica. La Habana: Científico-Técnica

Armas, K. E. G., Pérez, Y. G., y Rivero, A. F. M. (2017). LA TIPOLOGÍA TEXTUAL. CONCEPCIONES DIDÁCTICAS PARA LA COMPRENSIÓN. Revista Didasc@ lia: Didáctica y Educación. ISSN 22242643, 8(3), 137-150

Ávila, S. R. (2017). Aspectos discursivos y léxicos de la publicidad turística en medios impresos. Buscador de ideas afines y relacionadas. (s.f). Disponible en: http://www.ideasafines.com.ar/buscador-ideasrelacionadas.php

Bustos, J. M. (2009). Definición de glosarios léxicos del español: Niveles inicial e intermedio. Enseñanza \& Teaching: Revista Interuniversitaria de Didáctica, 19, 35-72

Cassany, D. (2007). Afilar el lapicero. Guía de redacción para profesionales. Barcelona: Anagrama

Castillo A., S. y Cabrerizo D., J. (2006). Formación del profesorado en educación superior. Didáctica y currículum. Volumen I. España: Editorial Mc Graw Hill

Córdova, C. A. (2014). Consideraciones sobre metodología de la investigación. Holguín: Universidad de Holguín. Centro de estudios sobre identidad cultural

Corrector ortográfico (2010). Disponible en:http://www. correctorortografico.com

Delis, Y. M. R., Campaña-Jiménez, R. L. y Gallego-Arrufat, M. J. (2018). Iniciativas para la adopción y uso de recursos educativos abiertos en Instituciones de Educación Superior. Educación Médica Superior, 32(4)

Diccionarios (s.f). Disponibles en: http://www. wikilengua.org/index.php/Lista_de_diccionarios_ en_Internet

Dubsky, J. (1983). Introducción a la estilística de la lengua. Santiago: Universidad de Oriente. Serie Humanidades. No. 4, 49-75

Estévez, M. (2006). Los métodos de investigación. En La investigación científica en la actividad física: su metodología (pp. 191 - 217). La Habana: Deportes 
Expresiones coloquiales (s.f). Disponible en: http:// www.coloquialmente.com/es

Gené, E., Olmedo, L., Pascual, M., Azagra, R., Elorduy, M., y Virumbrales, M. (2018). Evaluación de competencias en comunicación clínica en estudiantes de medicina con paciente simulado. Revista médica de Chile, 146(2), 160-167

Grass, E. (2002). Textos y abordajes. La Habana: Pueblo y Educación

Hernández, R. C. (2011). Contribución al desarrollo de la identidad cultural, a través de la asignatura Computación. Tesis presentada en opción a la categoría académica de Master en Educación Superior. Universidad de Holguín "Oscar Lucero Moya", Holguín

Hernández, R. C. (2012). El desarrollo de la identidad cultural desde el proceso de enseñanzaaprendizaje de la Computación. Tesis presentada en opción al grado científico de Doctor en Ciencias Pedagógicas. Universidad de Holguín "Oscar Lucero Moya", Holguín

Hernández, R. C. e Infante, M. E. (2018). Las técnicas de consenso en la investigación científica: reflexiones teórico-prácticas. Quito: Jurídica del Ecuador

Hernández, R. C., Hernández, S. L. y Terrazas, T. I. (2015). El desarrollo de la competencia ortográfica: una aproximación desde la práctica. Prefacio de Infante Miranda, M.E. Quito: Mendieta

Hernández, R. C., Infante, M. E. y Córdova, C. A. (2016). La identidad cultural y la Informática: retos al docente en la contemporaneidad. Quito: Mendieta

López, A. (2004). Diccionario enciclopédico de ciencias de la documentación. Madrid: Síntesis

López, R., y Eliza, A. (2018). Monitoreo, acompañamiento y la evaluación para mejorar la práctica docente en la competencia lee textos escritos en su lengua materna del área de comunicación del III Ciclo de Educación Básica Regular de la Institución Educativa $\mathrm{N}^{\circ}$ 80130 "Emérita Herrera Vega" del Distrito de Huamachuco-Provincia de Sánchez Carrión-Ugel Sánchez Carrión-La Libertad

Microsoft (2010). Suite ofimática OpenOffice. Disponible en: http://www.openoffice.org/es/

Microsoft. (Ed.) (2001). Diccionario de informática e internet España: McGraw-Hill Interamericana
Peña, R. (2013). Uso de las TIC en la vida diaria. México: Alfaomega

Pérez, J.A. (1999). Diccionario urgente de estilo científico del español. Disponible en: http://www. dlsi.ua.es/ japerez/pub/pdf/duece.pdf)

RAE Gestion (2016). Diccionario de la lengua española (Version 1.0): Real Academia Española (RAE) y la Asociación de Academias de la Lengua Española (ASALE)

Real Academia Española (2014). Diccionario de la lengua española (23. ${ }^{a}$ edición). Disponible en: http://drae.rae.es

Real Academia Española (2016). Diccionario del español jurídico. Disponible en: http://dej.rae.es

Real Academia Española (2017). Diccionario Panhispánico de Dudas. Disponible en: http://www. rae.es/recursos/diccionarios/dpd

Rodríguez, L. (2009). Español para todos: temas y reflexiones. La Habana: Pueblo y Educación

Roméu, A. (2012). La comunicación en la ciencia. Revista Educación. No. 107, 21 - 27

Salazar, A. V. L. (2018). Métodos y Técnicas de Investigación. DÍKÊ. Revista de Investigación en Derecho, Criminología y Consultoría Jurídica, (21), 323-326

Vigara, A. M. (2001). Libro de estilo de ABC. Barcelona: Ariel. Disponible en: http://books.google.es/ books?id=A8uf4ym076YC\&

\section{Anexo 1 \\ Guía de entrevista}

\section{Objetivo:}

Obtener información de los estudiantes, seleccionados intencionalmente, de primer semestre de las carreras de: Ingeniería en Software, Licenciatura en Derecho y Licenciatura Administración de Empresas y Negocios, que se imparten en la Universidad Regional Autónoma de los Andes (UNIANDES), extensión Ibarra, Ecuador, durante el período octubre de 2017 a febrero de 2018, sobre su preparación para la elaboración del proyecto integrador, texto de carácter científico.

Lugar: sala "Aristóteles", de la Universidad Regional Autónoma de los Andes (UNIANDES), extensión Ibarra, Ecuador 
Fecha: en tres momentos del período académico octubre de 2017 a febrero de 2018, al inicio, en la parte central de dicho período y al finalizar.

Aspectos tomados en cuenta en la primera entrevista:

- Objetivo que persigue la realización del proyecto integrador del nivel.

- Conocimiento sobre las temáticas a abordar y su relevancia.

- Alcance de la investigación y el contexto de desarrollo.

Aspectos tomados en cuenta en la segunda entrevista:
- Estructura textual establecida para el proyecto integrador.

- Integración de los aspectos de la investigación en desarrollo y el contenido de las materias que reciben.

Aspectos tomados en cuenta en la tercera entrevista:

- Organización y coherencia de la información recopilada, tanto teórica como práctica.

- Adecuación para cumplir con las exigencias formales y conceptuales de la presentación del informe.

- Nivel de dominio en las normas de la redacción científica para la textualización del proyecto.

Anexo 2

Rúbrica para revisión textual del proyecto integrador

NOMBRE:

MATRÍCULA:

\begin{tabular}{|c|c|c|c|}
\hline Criterios & Totalmente logrado & Medianamente logrado & Débilmente logrado \\
\hline Adecuación (4) & $\begin{array}{l}\text { El texto es organizado, respeta márgenes } \\
\text { y cumple con los criferios estandarizados } \\
\text { del proyecto integrador. (4) }\end{array}$ & $\begin{array}{l}\text { El texto es organizado, pero no respeta } \\
\text { márgenes ni se identifican los criterios } \\
\text { estandarizados del proyecto integrador. } \\
\text { (2) }\end{array}$ & $\begin{array}{l}\text { El texto es desorganizado } \\
\text { y no respeta márgenes ni } \\
\text { se identifican los criterios } \\
\text { estandarizados del proyecto } \\
\text { integrador (1) }\end{array}$ \\
\hline $\begin{array}{l}\text { Dominio del lenguaje } \\
\text { académico científico (3) }\end{array}$ & $\begin{array}{l}\text { El texto muestra objetividad, } \\
\text { impersonalidad, precisión en el } \\
\text { empleo de términos, predominio de lo } \\
\text { denotativo. (3) }\end{array}$ & $\begin{array}{l}\text { El texto muestra cierta subjetividad; } \\
\text { evidencia la primera persona del sujeto } \\
\text { enunciador; es medianamente preciso } \\
\text { en el empleo de términos y no hay total } \\
\text { dominio de lo denotativo. (2) }\end{array}$ & $\begin{array}{l}\text { El texto muestra subjetividad; } \\
\text { evidencia la primera persona del } \\
\text { sujeto enunciador; usa palabras } \\
\text { coloquiales y no hay total } \\
\text { dominio de lo denotativo. (1) }\end{array}$ \\
\hline Capacidad de síntesis (4) & $\begin{array}{l}\text { Se nota precisión en el tratamiento de } \\
\text { la información recopilada, tanto teórica } \\
\text { como práctica. } \\
\text { El texto muestra alta capacidad de } \\
\text { síntesis en el desarrollo del informe. (4) }\end{array}$ & $\begin{array}{l}\text { Se nota cierta dispersión en el tratamiento } \\
\text { de la información recopilada, tanto } \\
\text { teórica como práctica y presenta mediana } \\
\text { dificultad en relación al poder de síntesis } \\
\text { en el desarrollo del informe. (3) }\end{array}$ & $\begin{array}{l}\text { Presenta dispersión en el } \\
\text { tratamiento de la información } \\
\text { recopilada y evidencia } \\
\text { dificultad en relación al poder } \\
\text { de síntesis en el desarrollo del } \\
\text { informe. (2) }\end{array}$ \\
\hline $\begin{array}{l}\text { Coherencia } \\
\text { (4) }\end{array}$ & $\begin{array}{l}\text { Sus ideas son coherentes. Sus párrafos } \\
\text { presentan progresión del tema. Sabe } \\
\text { colocar un punto cuando acaba una idea. } \\
\text { (4) }\end{array}$ & $\begin{array}{l}\text { Sus ideas son coherentes, pero sus } \\
\text { párrafos no presentan progresión del } \\
\text { tema. No sabe colocar un punto cuando } \\
\text { acaba una idea. (3) }\end{array}$ & $\begin{array}{l}\text { Sus ideas no son coherentes y sus } \\
\text { párrafos no presentan progresión } \\
\text { del tema. No sabe colocar un } \\
\text { punto cuando acaba una idea. } \\
\text { (2) }\end{array}$ \\
\hline $\begin{array}{l}\text { Cohesión } \\
\text { (4) }\end{array}$ & $\begin{array}{l}\text { Utiliza adecuadamente la sustitución } \\
\text { lexical (pronombres, sinónimos, } \\
\text { hiperónimos), es decir, no repite los } \\
\text { términos. Hace un uso adecuado de } \\
\text { conectores que logran el sentido lógico } \\
\text { del texto. (4) }\end{array}$ & $\begin{array}{l}\text { Utiliza adecuadamente la sustitución } \\
\text { lexical (pronombres, sinónimos, } \\
\text { hiperónimos, es decir, no repite los } \\
\text { términos), pero no hace un uso adecuado } \\
\text { de conectores que logran el sentido } \\
\text { lógico del texto. (3) }\end{array}$ & $\begin{array}{l}\text { No utiliza adecuadamente la } \\
\text { sustitución lexical y no hace un } \\
\text { uso adecuado de conectores. (2) }\end{array}$ \\
\hline $\begin{array}{l}\text { Gramaticalidad } \\
\text { (4) }\end{array}$ & $\begin{array}{l}\text { Domina correctamente la acentuación } \\
\text { de las palabras; utiliza adecuadamente } \\
\text { los signos de puntuación. No comete } \\
\text { errores en la escritura de letras. } \\
\text { (4 errores máximos en todo el texto en } \\
\text { la acentuación o puntuación o alguna } \\
\text { falta leve). (4) }\end{array}$ & $\begin{array}{l}\text { Domina medianamente la acentuación de } \\
\text { las palabras y los signos de puntuación. } \\
\text { Comete algunos errores en la escritura de } \\
\text { letras. } \\
\text { (9 errores máximos en todo el texto en } \\
\text { la acentuación o puntuación o alguna } \\
\text { falta). (3) }\end{array}$ & $\begin{array}{l}\text { No domina la acentuación de } \\
\text { las palabras ni los signos de } \\
\text { puntuación. Comete varios } \\
\text { errores en la escritura de letras. } \\
\text { (10 errores en adelante en } \\
\text { acentuación o puntuación y } \\
\text { varias faltas). (2) }\end{array}$ \\
\hline $\begin{array}{l}\text { Uso de citas } \\
\text { (2) }\end{array}$ & $\begin{array}{l}\text { Usa citas largas y cortas, directas y } \\
\text { parafraseadas de diversos autores. } \\
\text { Coloca debidamente las referencias al } \\
\text { concluir el texto. (2) }\end{array}$ & $\begin{array}{l}\text { Usa citas largas y cortas, directas y } \\
\text { parafraseadas, pero no hay variedad } \\
\text { de autores. Coloca las referencias al } \\
\text { concluir el texto con algunos errores. (1) }\end{array}$ & $\begin{array}{l}\text { No identifica todas las citas y no } \\
\text { se ajusta a lo solicitado. Coloca } \\
\text { las referencias al concluir el } \\
\text { texto, pero con errores. }(0.5)\end{array}$ \\
\hline
\end{tabular}

\section{Recomendaciones:}

